\title{
Disintegration of Light Atomic Nuclei by the Capture of Fast Neutrons
}

\author{
By Prof. Willtam D. Harkins, David M. Gans and Henry W. Newson
}

\begin{abstract}
ABOUT thirteen disintegrations of neon nuclei $A$ have been obtained in 3,200 pairs, and approximately 100 disintegrations of nitrogen nuclei in 7,600 pairs of photographs of a Wilson chamber through which neutrons were passing. The source of the neutrons consisted of beryllium powder intimately ground with a mixture of mesothorium and thorium$\mathrm{X}$. The neutron source used was on the average more powerful in the experiments with neon than with nitrogen. If all the factors are taken into account, it is found that with identical atomic concentrations of neon and of nitrogen in the chamber, the neon nuclei are disintegrated much less often than those of nitrogen.
\end{abstract}

The average energies of the neutrons which have been found to disintegrate light nuclei are, in millions of electron volts, $5 \cdot 8$ for nitrogen, $7 \cdot 0$ for oxygen, and $11 \cdot 6$ for neon. Here the value for oxygen is taken from the work of Feather. The mass data indicate that the energy needed to supply mass increases in just this order, and is respectively $-1.4 \times 10^{6}, 0$ and $+2 \times 10^{6}$ electron volts, if the mass of the neutron is assumed to be that given by Chadwick, 1.0067, which is probably too high. Obviously the value assumed does not affect the differences between the energy values.

In a gas, ethylene, which consists of hydrogen and carbon, three disintegrations were obtained in 3,200 pairs of photographs. If carbon (12) is disintegrated by capture of the neutron the reaction is

$$
\begin{gathered}
\mathrm{C}^{12}+n^{1} \rightarrow \mathrm{C}^{13} \rightarrow \mathrm{Be}^{9}+\underset{\mathrm{He}^{4}}{\rightarrow} \\
12 \cdot 0036+1.0067 \stackrel{0155+4.002 \mathrm{I} 6}{\longrightarrow}
\end{gathered}
$$

or, if the mass assumed for the neutron is correct, $\Delta m=0.0074$, which is equivalent to $6.9 \times 10^{6}$ electron volts.

This corresponds to a velocity of $3.6 \times 10^{9} \mathrm{~cm}$. per sec., so only neutrons of a velocity higher than this should be effective in disintegrating carbon of mass 12. The smallness of the yield of disintegrations which we have obtained with carbon is thus to be expected, especially since probably less than one- fifth of the neutrons have velocities higher than this.

A remarkable relation which has been found to hold without exception is : in disintegrations by capture of a neutron the kinetic energy almost always decreases, is sometimes conserved, but in no case increases.

It has been pointed out previously by Harkins that the values for the energy which disappears suggest definite energy values for the $\gamma$-rays into which this energy is converted, but the accuracy of the work is not yet sufficient to prove that this is true.

It may be assumed that the neutrons in the stars are scattered by the atomic nuclei and thus take part in the temperature distribution of velocities of the atoms. If the neutrons of higher velocity are captured much more often than those of lower velocity, the distribution will be affected. Our experiments give no information concerning the capture of neutrons without disintegration, but only for those cases in which the capture is revealed by the accompanying disintegration.

It is of interest in this connexion to consider the minimum energy of the neutron which has been found to give a disintegration. The values, in millions of electron volts, are 1.9 for nitrogen, and 7.8 for neon. The corresponding maximum values are $16 \cdot 0$ and $14 \cdot 5$, the lower maximum for neon being due to the smallness of the number of disintegrations which have been obtained in this gas. An energy of $1.9 \times 10^{6}$ corresponds to a mean temperature of the order of $10^{10}$ degrees, but at $10^{8}$ degrees a considerable number of neutrons should have this energy, and a moderate number even at $10^{7}$, so it is not unreasonable to suppose that nitrogen nuclei are disintegrated by this process in the stars.

A part of this work was presented by Harkins on June 23 at a symposium on nuclear disintegration under the auspices of the Century of Progress Exposition, Chicago. Other papers were presented by Cockroft, Lawrence and Tuve, and a general discussion of the theory was given by Bohr.

\section{Third International Congress for Experimental Cytology}

$\mathrm{T}$ HE Third International Congress for Experimental Cytology, which was held in Cambridge on August 21-26, under the presidency of Prof. Th. Huzella, of Budapest, was attended by more than two hundred members from all parts of the world. Some seventy papers were read, grouped under the headings of cell respiration and metabolism, electrophysiology, secretion and digestion, tissue culture, Entwicklungsmechanik, and virus cultivation.

One of the main impressions left by a most interesting Congress is the successful use of the technique of explantation and tissue culture by cytologists, embryologists, and medical research workers for the study of problems of the most diverse nature. This note was struck by Prof. Huzella, in his presidential address on tissue culture in relation to the problems of biology and medicine, and it constantly recurred in the papers read to the Congress. Another feature of modern biological research which was well brought out was the emphasis on the study of cell functions as they actually occur in vivo, rather than by examination of fixed and stained preparations, and the variety and ingenuity of the methods that have been evolved for this purpose. The proceedings also showed how wide a 'no-man's-land' there still is between experimental cytology, which is limited by the resolving power of the microscope, and biochemistry and biophysics, which deal with the phenomena of the cell on a molecular scale. It was a specially valuable feature of the Congress that cytologists and biochemists and biophysicists were able to learn one another's points of view, and to hear of some of the progress being made towards linking up physiological with physicochemical events. Lastly, mention must be made of the moving pictures, which showed what a valuable instrument of research is provided by the speeded-up micro-cinematograph film. 
In the discussion of tissue culture, Prof. Huzella described experiments on the effect of magnetic fields on heart cultures. His pupil, Dr. Julia Lengyel, showed that constant magnetic fields affect the intracellular substance, but have no effect on the cells themselves. Prof. Huzella dealt with the effect of intermittent magnetic fields. The cells are found to grow along the lines of force. Prof. Huzella attributes the effect to induced electric currents in the medium. In the discussion, the alternative explanation was suggested that the living cell is the seat of circulating electric currents, and so has a magnetic moment. These results link up with previous findings by Prof. Huzella and others that cells always tend to grow along lines of strain, whether caused by stretching, crystallisation in the culture medium, or in other ways.

Prof. J. de Haan and K. J. Feringa, of Groningen, have devised a method by which comparatively thick explants can be grown while being perfused with a physiological fluid. They were able to demonstrate the differentiation of wandering cells from a rabbit exudation into typical lymph tissue, and even the formation of non-nucleated cells resembling red blood corpuscles. Their experiments were illustrated by a cinematograph film.

Prof. C. C. Speidel, of Virginia, has also found the cinematograph of great value in studying the growth, irritation, and repair of nerves. He works on anæsthetised tadpoles, which are fixed under the microscope. The tip of the tail is cut off, and in the rapid process of regeneration the details of nerve outgrowth can be followed in vivo. By his remarkably clear and detailed observations, Prof. Speidel has been able to settle many doubtful points in neural physiology.

Dr. H. Okkels, of Copenhagen, described work on the dynamic cytology of the thyroid gland. When called into sudden activity, the cells undergo a cycle of changes. There is a phase of synthesis of the secretion, in which the mitochondria play the decisive part, and then a phase of discharge, which is governed by the so-called Golgi apparatus. Dr. E. S. Duthie, of Dublin, has found similar phenomena in the cells of various digestive glands.

In the discussion on virus growth, there was universal testimony to the value of tissue cultures as a means of virus propagation. Dr. G.H. Eagles, of London, indeed, maintained that viruses can be grown in cell-free media, and his paper provoked a long and interesting discussion. He agrees, however, that the presence of living cells is favourable to virus growth. Tissue cultures not only form a convenient substitute for animal propagation in the culture of viruses, but also, as was shown in a series of papers from different workers, allow of observations on cell pathology and immunity phenomena that would be impossible on the living animal.

In the field of Entwicklungsmechanik, there were a series of important communications. Dr. H. B. Fell, of Cambridge, has obtained differentiation of kneejoints in explants of chick limb-buds taken from the egg at a stage when no differentiation is visible. Dr. R. G. Canti showed a fascinating film of the process of differentiation in Miss Fell's preparations. Prof. R. Chambers, of Now York, has found that explants of mesonephric tubule segments are converted into closed tubules. When this stage is reached, phenol red accumulates in the lumina of the segments. This effect depends on the maintenance of the cell respiration, and is an instance of cells taking on their physiological functions in vitro.

J. Holtfreter, of Berlin, described work on the amphibian embryo, which he has succeeded in cultivating in vitro. C. $\mathrm{H}$. Waddington, of Cambridge, who has devised a method for keeping the warmblooded chick embryo alive out of the egg for a few days, also described the result of experimental interference with normal development. Both workers have been able to show the determining effect of the endoderm for differentiation in a number of beautiful experiments. Holtfreter, describing his work on the 'organiser', showed that it is present in all adult tissues, and in a latent form in the egg and in embryo tissues. In the latter, no organising power is shown until the tissue is boiled. Needham, Waddington and Needham have made it practically certain that the organiser is a chemical substance by obtaining neural tube formation in the embryo with petrol-ether extracts (see Nature of August 12, p. 239).

This demonstration of what may be called an intra-embryonic hormone is of great interest. Nevertheless, its ubiquitous occurrence tends to throw the emphasis on the innate properties and responses of the embryonic tissue, rather than on the chemical stimulus from without, as a determining factor in differentiation.

The present Congress differed from previous ones, in that the scope was widened to include cell respiration and metabolism and cell electrophysiology, to each of which a whole day was devoted. Prof. E. D. Adrian, of Cambridge, opened the discussion on electrophysiology, and papers were read on biological potentials and currents, and the mechanism of selective passage and concentration of ions by cell membranes. Dr. M. Dixon, of Cambridge, opened the discussion on cell respiration. Prof. A. SzentGyörgyi, of Szeged, read a provocative paper on non-enzymic catalysts of cellular oxidation. One useful feature of the day's discussion was the demonstration of the wide variety of oxidation mechanisms, and the differences in response to cyanides and other respiratory inhibitors, that occur in the different phyla of the plant and animal kingdom. This must have been especially valuable to the biochemists, who have perhaps confined their attention to too few kinds of organisms.

B. Woolf.

\section{Research in the British Non-Ferrous Metal Industries}

$\mathrm{T}$ $\mathrm{HE}$ annual meeting of the British Non-Ferrous Metals Research Association was held at Birmingham on June 19, and the report for the past year shows that, in spite of the difficulty of the times, the expenditure on research has been slightly increased to $£ 17,000$ a year.

Brighter homes should resuit from the choice of suitable metals and alloys arising from the study which is being made of the resistance to tarnishing of polished surfaces of metals used for finger plates, door knobs, shop fronts and the like. Much work has also been done on an investigation dealing with the factors involved in the bursting of water pipes due to frost, concerning which little is at present known. The mechanism of ice formation and the behaviour of freezing water in pipes of different 